\title{
BMJ Open Optimum hospice at home services for end-of-life care: protocol of a mixed- methods study employing realist evaluation
}

\author{
Claire Butler, ${ }^{1}$ Charlotte Brigden, ${ }^{2}$ Heather Gage, ${ }^{3}$ Peter Williams, ${ }^{4}$ \\ Laura Holdsworth, ${ }^{5}$ Kay Greene, ${ }^{6}$ Bee Wee, ${ }^{7}$ Stephen Barclay, ${ }^{8}$ Patricia Wilson ${ }^{1}$
}

To cite: Butler C, Brigden C, Gage $\mathrm{H}$, et al. Optimum hospice at home services for end-of-life care: protocol of a mixedmethods study employing realist evaluation. BMJ Open 2018;8:e21192. doi:10.1136/ bmjopen-2017-021192

- Prepublication history for this paper is available online. To view these files, please visit the journal online (http://dx.doi org/10.1136/bmjopen-2017021192).

Received 14 December 2017 Revised 12 February 2018 Accepted 20 March 2018

\section{Check for updates}

${ }^{1}$ Centre for Health Services Studies, University of Kent, Canterbury, UK

${ }^{2}$ Pilgrims Hospices in East Kent, Canterbury, UK

${ }^{3}$ Department of Clinical and Experimental Medicine, University of Surrey, Guildford, UK

${ }^{4}$ Department of Mathematics, University of Surrey, Guildford, UK

${ }^{5}$ Primary Care and Population Health, Stanford School of Medicine, Stanford, California, USA

${ }^{6}$ National Association for Hospice at Home, Fareham, UK ${ }^{7} \mathrm{NHS}$ England, Wakefield, UK ${ }^{8}$ Primary Care Unit, Department of Public Health and Primary Care, University of Cambridge, Cambridge, UK

\section{Correspondence to} Professor Claire Butler; c.butler-779@kent.ac.uk, clairesinnott@aol.com

\section{ABSTRACT}

Introduction Hospice at home (HAH) services aim to enable patients to be cared for and die in their place of choice, if that is at home, and to achieve a 'good death'. There is a considerable range of $\mathrm{HAH}$ services operating in England. The published evidence focuses on evaluations of individual services which vary considerably, and there is a lack of consistency in terms of the outcome measures reported. The evidence, therefore, does not provide generalisable information, so the question 'What are the features of hospice at home service models that work, for whom, and under what circumstances?' remains unanswered. The study aims to answer this question. Methods and analysis This is a mixed-methods study in three phases informed by realist evaluation methodology. All HAH services in England will be invited to participate in a telephone survey to enable the development of a typology of services. In the second phase, case study sites representing the different service types will collect patient data and recruit carers, service managers and commissioners to gather quantitative and qualitative data about service provision and outcomes. A third phase will synthesise and refine the results through consensus workshops.

Ethics and dissemination The first survey phase has university ethics approval and the second phase, Integrated Research Application System (IRAS) and Health Research Authority (HRA) approval (IRAS ID:205986, REC:17/L0/0880); the third phase does not require ethics approval. Dissemination will be facilitated by project coapplicants with established connections to national policy-making forums, in addition to publications, conference presentations and reports targeted to service providers and commissioners.

\section{INTRODUCTION}

Hospice at home (HAH) services have evolved in England since the development of the 'modern hospice movement' in the late 1960s. These services tend to share the following characteristics:

- Aim to enable patients to be cared for and die in their place of choice, if that is their own home.
Strengths and limitations of this study

- The realist evaluation approach enables the complexity of different hospice at home models to be 'unpacked' within their context to understand what features work for whom and in what circumstances.

- Involving palliative patients at the end of life and their carers will be challenging in relation to approaching participants at this sensitive time and potential loss to follow-up of carers who are subsequently bereaved.

- In-depth case studies of up to six hospice at home models with different features will be part of the realist evaluation, therefore it is possible that not all feature combinations identified in the model typology from the survey of hospice at home services will be included.

- Patients without an informal carer involved on a daily basis will be excluded from the study.

- Patients and carers who are unable to communicate in English will be excluded from the study.

- Employ 'specialist' staff with high levels of palliative care experience.

- Ability to provide more staff time with the patient than pre-existing/other services.

A review of the literature identified service development projects and evaluations of services in England that have HAH characteristics. ${ }^{1-31}$ Each study focused on an individual service and used various methods to investigate locally determined patient, carer and professional outcomes. Outcomes frequently focused on one or more of the following: place of death, fulfilment of wishes, carer satisfaction, carer bereavement, symptom management, experience of the service, hospital admission.

The literature mirrors the fact that different HAH services have grown up in an ad hoc fashion resulting in a considerable range of HAH services in terms of operation, staffing and function, making it difficult to identify 
similar services in comparable settings. There has been little consensus as to what standards characterise such a service or what makes a service more or less effective. The National Association for Hospice at Home (NAHH) has recommended six core, national standards for HAH services developed through three national HAH stakeholder workshops held in 2011-2012. ${ }^{32}$ The NAHH also worked with Hospice UK and conducted a survey across 76 HAH services in England which provided some useful data to start to describe the landscape of HAH services. This survey confirmed that more than one model of HAH service exists, and they are not homogeneous in their activities or outcomes. ${ }^{33}$

The best way to provide care within a patient's home and how this can be maintained for as long as possible was identified as one of the top 10 research priorities (in the UK) in a James Lind Alliance priority-setting partnership on palliative and end-of-life care published in January 2015. ${ }^{34}$ It has been recognised that most people have a preference to die at home ${ }^{35}$ and indeed the number of patients wishing to die at home is increasing. ${ }^{36-38} \mathrm{~A}$ cost analysis from one study found that users of the HAH service had significantly lower utilisation of hospital services. ${ }^{24}$ Given that almost half of annual deaths in England take place in National Health Service (NHS) hospitals, ${ }^{39}$ it seems there is potential to increase the number of patients accessing community care and at the same time reduce NHS acute care costs. Demographic studies predict a future of increasing numbers of older people and increasing numbers of deaths. ${ }^{39}$ A recent Health Ombudsman report highlighted how more needs to be done to support the health service in delivering quality care at the end of life. ${ }^{40}$ HAH services offer an acceptable solution to meet these social and political drivers, yet their expansion has so far been haphazard. It is therefore important to understand how best to deliver effective HAH services at scale and in a cost-effective manner to achieve the outcomes desired.

No study comparing different types of HAH services has been identified. The variation in services and the settings in which they operate makes it difficult to conduct traditional comparative analyses and to achieve a meaningful synthesis of evidence which would help inform future service development and planning. This paper presents the protocol of a funded study running from 1 February 2017 to 31 January 2020 which aims to fill this evidence gap.

\section{Aims and objectives}

The study's aim is to investigate the impact of the organisation and delivery of different models of $\mathrm{HAH}$ on patient and carer outcomes and experiences of end-oflife care from the perspective of service users, their family carers, service providers and commissioners. Our overarching research question is: What are the features of HAH service models that work, for whom and under what circumstances?
Objectives to address the primary research question are as follows:

1. Identify the range and variation of HAH models operating across England.

2. Categorise the models by type, setting and key features.

3. Select case studies of each model to enable an assessment of the impact of that model on patient and carer outcomes.

4. Investigate the resource implications and costs of patient care in each model.

5. Explore the experiences of patients, family carers, providers and commissioners of the different HAH models.

6. Identify the enablers and barriers to embedding HAH models as part of service delivery for end-of-life care.

HAH is a complex intervention and part of a whole system of health and social care delivery. The research design is informed by realist evaluation, ${ }^{41} 42$ a theory-driven methodology increasingly used to evaluate complex interventions ${ }^{43}$ including services for end-of-life care. ${ }^{44}$ At the core of realist evaluation is the notion of 'generative mechanisms'; a generative mechanism is a causal link, the black box which leads from A to B and creates an 'effect'. ${ }^{45}$ Realist evaluation theorises what the mechanisms are, the relationship between mechanisms, the context in which they are operating and the effects they produce through propositions, which take on a basic formula of: context+mechanism=outcome (CMO). The aim is to identify patterns to support an explanatory theory about what mechanisms are working (or not) in a given situation. ${ }^{46}$ Data are sought to prove, refute and ultimately refine the conjectured CMO configurations.

\section{METHODS AND ANALYSIS}

The method comprises three phases:

\section{Phase 1: telephone survey}

A national telephone survey of all adult HAH services in England. There were 127 services identified from the Hospice UK Service Directory, cross-referenced with the NAHH database (received 28/10/2016) and contact will be made with service managers. The purpose of the survey is to produce a comprehensive map of the range and variation of HAH services and to group them into service model types, sharing similar characteristics. The survey will elicit information on service setting, configuration, operations and activity.

The interpretation of the survey findings will involve iterative consensus work with the project steering group and public and patient involvement advisory group to develop model types from the survey information. Categorical variables (eg, urban/rural, presence of hospice building(s), involvement of registered nurses (Yes/No), $24 / 7$ care (Yes/No) etc) will be cross-tabulated with each other in order to identify underlying associations. Continuous variables (eg, area population, area (square miles), 
number of individuals employed by the service) will be compared between different categories of each categorical variable, as well as being plotted against each other, in order to identify underlying associations. These results will assist in identifying natural groupings.

From this work, it is envisaged that at least four highlevel types of HAH services will be distinguishable. This estimate is based on previous survey work which indicated there to be at least two types of model ${ }^{33}$ and an earlier literature review ${ }^{47}$ in which we have found that there are services with and without registered nursing provision and services with and without the availability of rapid access 24/7.

\section{Phase 2: case studies}

A sampling framework will be used to purposively select up to six case studies of HAH services from the highlevel types identified in phase 1. Each type of model will be represented by at least one case study and the HAH service will be the unit of analysis. The approach will employ mixed methods to gain in-depth understanding of the impact of each type of model. This design also allows methodological flexibility to generate theoretical insights from the findings, ${ }^{48}$ which is a key requirement for realist evaluative design. ${ }^{42}$ The case studies will be used as test beds for candidate CMO configurations. ${ }^{49}$ These candidate CMO configurations will be identified through our systematic mapping of the literature, our previous completed research and the NAHH core standards for HAH services. ${ }^{32}$ We will also use normalisation process theory (NPT) ${ }^{50}$ as a middle-range theory to understand how each HAH model becomes embedded within a whole system of care. NPT is focused on the 'work' that is involved in implementing a service, such as how staff make sense of the work they do and how they reflect on their practice. ${ }^{51}$ NPT has previously been used successfully within an overall realist evaluative design to understand in detail the enabling contexts within CMO configurations. ${ }^{52}$

The impact of the HAH service on the following outcomes will be evaluated:

- The quality of death, using the quality of death and dying (QODD) tool, ${ }^{53-55}$ completed with bereaved carer from 4 months post death, over the telephone; this is the primary outcome measure.

- Overall experience of care and support, using two questions selected from the National Survey of Bereaved People ${ }^{56}$ and through in-depth interviews with a subset of family carers post bereavement.

- Whole system resource use gathered through interviews with carers every 2 weeks from recruitment to patient death using a customised version of the Ambulatory and Home Care Record (AHCR) ${ }^{57}$ from which costs will be calculated.

- Service provider and commissioner views about enablers and barriers to delivering the HAH model, gathered through qualitative interviews. The service manager interviews will include items relevant to understanding the economic costs of running each case study HAH service.

In addition, the Integrated Palliative Care Outcome Scale tool, ${ }^{58}$ the Australia-modified Karnofsky performance scale and the phase of illness ${ }^{59}$ will be completed by care professionals on entry to the HAH service to facilitate an understanding of the casemix of each service.

\section{Recruitment and informed consent}

The study comes under the remit of the Mental Capacity Act 2005. Patients and their main lay carer within the case study sites will be invited to participate in the study when they are admitted to the HAH service. Service staff will introduce the study to the patient and their carer, provide written information and gain their consent if they agree to participate. Due to the nature of the patient population, who will be close to the end of life, it is anticipated that some of the potential participants will be unable to provide informed consent (eg, as a result of impaired cognition or impaired consciousness). For this reason, a variable consenting process, involving consultee assent, will be used. This method is increasingly used and accepted by NHS ethics committees as a process for gaining consent for patients who lack capacity. ${ }^{60} 61$ If the patient is deemed not to have capacity, then a personal consultee (ie, 'someone who has a role in caring for the person who lacks capacity or is interested in that person's welfare but is not doing so for remuneration or acting in a professional capacity') will be approached for advice regarding the patient entering the study. The personal consultee could be a relation or a friend of the patient. The personal consultee will be given written information about the study and asked whether in their opinion the patient would have any objection to taking part. If the patient is deemed not to have capacity, and no personal consultee is available at the time or willing to take the responsibility, then a nominated consultee will be approached for advice regarding the patient entering the study; this is usually a health professional who knows the patient and is independent of the research team.

\section{Sample size}

\section{Quantitative data collection sample size}

The scores for the primary outcome measure, the QODD, range from 0 to 100 . Hales $e$ t $a t^{62}$ identified 30 and 70 as cut-offs for distinguishing terrible/poor, intermediate and good/almost perfect quality of death. On the basis of a difference of 10 points representing a meaningful change, and using an SD of $16.41,{ }^{63}$ at least 44 participants in each model would be required for comparisons between any pair. To allow for participant drop-out of $33 \%$, the required sample size is 66 patients per model type. The drop-out rate is based on a prospective trial of an intervention which followed up with the carers of patients involved who were sent the 24-item intensive care unit QODD questionnaire 4-6 months post death. They received a $55.4 \%$ response rate and it is predicted that contact through bereavement services and the telephone 
interview approach will achieve a better response than the postal survey approach used in that study. ${ }^{64}$

\section{Qualitative data collection sample sizes}

Using a purposive sampling approach, ${ }^{65} 5-10$ managers, healthcare staff and commissioners in each case study site will be interviewed.

It is anticipated that up to 20 bereaved family carers per site will be interviewed until data saturation is reached to explore experiences of service use, particularly what aspects of service provision contribute to positive and negative experiences of care in order to understand what aspects of care are most valued by service users.

\section{Data analysis}

\section{Quantitative data analysis}

The characteristics of patients in the different service models will be summarised using relevant descriptive statistics (proportions, medians, ranges, means, SDs, 95\% CIs, etc) before being compared on the basis of each patient's sociodemographic, clinical and carer features using the appropriate bivariate test (including one-way analysis of variance, $\mathrm{X}^{2}$ and Kruskal-Wallis tests, depending on the nature of the variable). Exploratory regression modelling will be used in order to investigate the effect of each service model on the primary outcome (QODD), controlling for sociodemographic, clinical and carer features. Stepwise regression methods (backward elimination approach, commencing with a set of covariates which have been agreed on as important by the research team) will be used. The fitted parameters in the final models, along with their significance, will indicate if service type is associated with differences in QODD scores.

\section{Economic analysis}

The economic analysis will be at two levels:

First, a descriptive analysis will be conducted of the resources and costs of running each case study $\mathrm{HAH}$ service. Information on staffing and activity rates will enable costs per patient receiving HAH to be calculated.

Second, a patient-level analysis will be undertaken. Whole system resource use in the end-of-life care will be captured prospectively from the point of recruitment to the study for each patient using a customised version of the AHCR, administered by telephone to the carer every 2 weeks until death. Retrospective data will also be collected from the carer shortly after recruitment to cover the period before the HAH service started. Service utilisation data will cover primary, community, hospital, hospice, social care, voluntary and informal care received. The AHCR has been developed and widely used for assessing resource use in home palliative care in Canada, including carer burden. In a recent systematic review of approaches to capturing the financial costs of family care giving within a palliative care context, the AHCR was identified as the only validated tool covering formal and informal services. ${ }^{66}$ The AHCR has been recently piloted in England by the research team and found to be both acceptable to participants and sensitive. Experience in the pilot indicated how the AHCR will be customised for use in the British NHS context.

Service use data, once captured, will be grouped into 4-6 time periods of approximately equal sample size, delimited by survival time from the start of service use data collection. The cut points will be determined by the distribution of the data. In the research team's previous study, $6 \%$ of patients referred to a HAH service had died within 2 days, $40 \%$ within 1 month, $62 \%$ within 2 months and the remaining $38 \%$ were referred over 2 months before death. Resource use will be converted to costs using national tariffs. ${ }^{67}$ Informal care will be valued using replacement cost methods.

For each of the models of HAH service provision, an average cost per day of treatment will be estimated for the 4-6 time periods. Costs will be presented as means and median, given the typical skew in the distribution of costs. Comparison of costs between HAH models will be assessed for significance using the Mann-Whitney U test. Sensitivity analysis for costs will be handled deterministically, varying the amount of resource use between their upper and lower limits for each HAH model. Costs will be analysed in relation to outcomes from different models in a cost-consequences framework.

\section{Qualitative data analysis}

Interviews will be transcribed and uploaded into NVivo to assist with data management and analysis. Analysis will be iterative with the aim of testing and refining programme theories and further developing provisional CMO configurations. ${ }^{42}$ As described above, NPT will be used to understand why a model has or has not been embedded within a whole system of care, and burden of treatment will be used to understand the impact of the model on patients and carers. NPT offers a well-established framework for analysis in order to understand implementation processes through the perspectives of multiple stakeholders, including: service users, service providers and commissioners. ${ }^{68}$ Constructs from the NPT framework will form the basis of a deductive coding structure. Analysis will also seek to identify any emergent themes not covered by NPT. Synthesis of an NPT informed coding framework alongside an inductive approach ${ }^{69}$ allows for a focused and yet open qualitative approach that allows unexpected findings to emerge. ${ }^{68}$

\section{Phase 3: stakeholder consensus}

The final phase comprises two national consensus workshops, with up to 60 participants attending each workshop. Participants will be identified through the NAHH and our project steering group. Stakeholders will include service providers, commissioners and service user representatives. The purpose of the workshops is to validate interpretation of the data and to refine our understanding of the specific features of HAH models that work, for whom and under what circumstances. 
Emerging findings and relationships between CMOs will be presented to stakeholders. ${ }^{42}$ The explicit aim of the workshops will be to refine CMO configurations and develop consensus on what type of HAH services are likely to work best and in what circumstances. The workshops will also contribute to translating findings into information that is relevant to managers and commissioners of HAH services. Consensus workshop methods will be used ${ }^{70}$ to facilitate discussion. Detailed notes of the discussions will be written and used to verify or challenge CMO configurations. Participants will be sent a workshop report and have the opportunity to comment on the study conclusions.

\section{Synthesis}

Data analysis from each phase of the study will be synthesised through a realist evaluative process comprising four stages $^{71}$ :

\section{Stage 1}

Articulation of programme theories and propositions from literature review already undertaken, stakeholder insight (study steering group and service users) and phase 1 of the study (national telephone survey). Identification of candidate CMO configurations.

\section{Stage 2}

Data collection from the model case studies in phase 2 of the study, to test and refine propositions.

\section{Stage 3}

Map the outcomes of each model including costs; interrogate what contexts and mechanisms explain the pattern of outcomes.

\section{Stage 4}

Through stakeholder consensus (phase 3 of the study), refine explanatory $\mathrm{CMO}$ configurations to evaluate what HAH model works best, for whom and in what circumstances.

\section{Patient and public involvement}

A lay advisory group was set up through a local hospice to inform the development of the study design, including feedback on the project idea, research question and outcome measures, reviewing funding application drafts and the plain English summary. The group consisted of four members, including two bereaved carers and two members of the public. The bereaved carers had previously had direct experience of HAH service as carers for patients receiving the service. The members of the public (one being a hospice volunteer) had a keen interest in research and the work of the hospice.

Membership of the group to support the study development had been advertised to carers, patients and members of the public locally but no patients came forward. This was not unexpected as experience from a previous study showed that palliative patients found it difficult to participate continuously due to ill health. Involvement from the bereaved carers was key, as carers are the main participants in the study, and these group members went on to become part of the research team as lay coapplicants on the research grant application.

The lay advisory group members were involved in the design of the study through face-to-face meetings and one-to-one via email. They influenced the design of the study, and for phase two in particular, they were able to feedback on the appropriateness of the data collection tools and what procedures should be in place when approaching and involving patients, carers and bereaved carers in the research, at a vulnerable time on a sensitive topic. Examples of their input are: the inclusion of additional support from research staff to help carers with the completion of the service use information and the provision of information for carers to access further advice and support from the hospice should they need it following the completion of the QODD questionnaire and interview. During the course of the project the coapplicant members will be invited to provide feedback on the design of study materials, including information sheets, study leaflet and study outputs, such as lay summaries to disseminate the results to study participants. As patients who have capacity will be invited to participate, feedback on the patient version of the information sheet will be sought from current hospice patients through the hospice day-care service. The lay coapplicants will be invited to input on decision-making as stakeholders in the consensus of the CMO configurations for the realist evaluation. Appropriate training will be provided by the University of Kent and the lay coapplicants will receive payment to cover their time and expenses. All members of the research team work with the lay coapplicants and advisory group and in addition, the role of one member of the team is focused on coordinating and facilitating patient and public involvement to ensure it is threaded throughout the project.

\section{ETHICS AND DISSEMINATION}

The study has been designed so that the burden to patients will be minimal, requiring consent to the collection of data already recorded for clinical purposes and consent to invite their carer to participate.

The main burden will fall on informal carers, to collect service utilisation data and then to respond to the telephone questionnaires administered postbereavement. Those who agree in addition to participate in a qualitative interview will be consented again and may find the interview process emotional and an opportunity to express their views about their experiences. The following steps have been taken to mitigate the burden for carers: the postbereavement data will be collected at 4 months; research staff will be trained and supported by the chief investigator, an experienced palliative care clinician and will be sensitive to the feelings of participants; interviews will be terminated if significant signs of distress develop and there is a distress protocol to provide support and follow-up. 
Phase 1 approvals: NHS Health Research Authority approval reference 17/HRA/0299. SRC ethics panel of the University of Kent School of Social Policy, Sociology and Social Research, approval 13/12/16.

Phase 2 approvals: National Research Ethics Service, London-Queen Square Research Ethics Committee, IRAS 205986; REC:17/LO/0880.

Phase 3: Interpretation and dissemination of findings, ethics approval not required.

\section{Safety considerations}

Research interviews may involve lone workers undertaking interviews at a carer's home. There are standard university policies and procedures for such situations which will be followed.

\section{Data deposition and curation}

Throughout the study we will be fully compliant with the provisions of the Data Protection Act 1998, Human Rights Act 1998, NHS Code of Practice on Confidentiality and Common Law of Confidentiality. Participants' confidentiality will be ensured by using unique, untraceable identification code numbers to correspond to electronic data in the computer files.

All names will be anonymised and will be stored as a password-protected electronic file exclusively on University of Kent PCs/servers. Passwords will be restricted to: the chief investigator, the project manager and research personnel who directly engage with participants for the purposes of collecting data.

\section{Dissemination plan}

Our primary output will be guidelines for services and commissioners to guide resource allocation and service development of HAH services. The guidelines will show what models/features of HAH services work best and at what cost and enable providers and commissioners to identify what the optimum HAH service model or key features of a HAH service would be for their population in their locality and organisational systems. The format of this guidance will be informed as part of the consensus workshops in phase 3 of the study. Additionally, the consensus events themselves will offer the opportunity for service providers to come together to share challenges and discuss good practice.

\section{Policy-maker, commissioner and professional engagement}

We aim to reach commissioners, palliative care service providers and a wider professional audience through strong coapplicant links with existing forums (eg, the Commissioning Assembly, the NAHH, Hospice UK) and through publication in health services journals and conference presentations.

\section{Written publications}

The full and complete account of the research will be published in the NIHR HS\&DR Journal; this will allow the research to be freely and publicly available via the NIHR journals library website. We also aim to publish in peer-reviewed journals to reach broad audience coverage in community services as well as palliative care services.

A plain English summary for public and patient engagement and dissemination will be written and this will also be available to our research participants.

\section{Presentations}

Oral presentations will be submitted to existing research forums such as the European Association of Palliative Care Congress; Clinical Research Network forums; Cicely Saunders Institute, King's College London; Hospice UK annual conference; NAHH conference.

\section{Social media}

We will use twitter (\#opelstudy) throughout the project via the Centre for Health Services Studies twitter account (@CHSS_Kent) to update on progress and debate, including discussions at the consensus event.

\section{Public}

Dissemination of findings aimed at the public will be facilitated through links with organisations including the National Council for Palliative Care and Dying Matters.

Contributors CBu prepared this manuscript, led the research team and supervised the development of the protocol. CBr undertook the literature review and coordinated the writing of the protocol. HG devised the economic evaluation methodology. PW devised the statistics methodology. KG provided advice and context about hospice at home services in England to enable the development of the methodology. BW supported the development of the dissemination strategy. SB supported the development of the methodology from a primary care clinical perspective with his experience of previous research in this area. PW proposed the realist evaluation methodology and with LH devised the qualitative data methodology.

Funding The study has been funded by the Health Services and Delivery Research programme of the National Institute for Health Research (Reference 14/197/44).

Disclaimer We attest that we have obtained appropriate permissions and paid any required fees for use of copyright protected materials. The views and opinions expressed therein are those of the authors and do not necessarily reflect those of the HS\&DR Programme, NIHR, NHS or the Department of Health.

Competing interests None declared.

Patient consent Not required.

Ethics approval NRES Committee, London Queens Square.

Provenance and peer review Not commissioned; externally peer reviewed.

Author note All authors have signed off on the study protocol.

Open Access This is an Open Access article distributed in accordance with the terms of the Creative Commons Attribution (CC BY 4.0) license, which permits others to distribute, remix, adapt and build upon this work, for commercial use, provided the original work is properly cited. See: http://creativecommons.org/ licenses/by/4.0/

(C) Article author(s) (or their employer(s) unless otherwise stated in the text of the article) 2018. All rights reserved. No commercial use is permitted unless otherwise expressly granted.

\section{REFERENCES}

1. Addington-Hall J, Gerard K, Brien S, et al. Variations in 'Out of hours' end of life care service provision across primary care organisations in England and Scotland: NIHR SDO programme project report, 2013.

2. Blanchard $\mathrm{H}$. An evaluation of a hospice at home service: a questionnaire survey of GP \& district nurse referrers. BMJ Support Palliat Care 2011;1:264-73. 
3. Buck J, Moth L, Webb L, et al. Hospice at home: optimising care and enabling home death. BMJ Support Palliat Care 2014;4(Suppl 1):A44.2-A44.

4. Butler C, Holdsworth LM, Coulton S, et al. Evaluation of a hospice rapid response community service: a controlled evaluation. BMC Palliat Care 2012;11:11.

5. Campbell M, Grande G, Wilson C, et al. Exploring differences in referrals to a hospice at home service in two socio-economically distinct areas of Manchester, UK. Palliat Med 2010;24:403-9.

6. Clayton B, Spencer L. Help the hospices poster presentations: $\mathrm{P} 10$ hospice rapid response service - a prospective analysis. BMJ Support Palliat Care 2013;3:A13.

7. Davis C. End of life care: a rapid response hospice at home service. Nurs Older People 2010;22:22-4.

8. Exley C, Tyrer F. Bereaved carers' views of a hospice at home service. Int J Palliat Nurs 2005;11:242-6.

9. Gibson V, McEvilly L, Standfield R. Help the hospices poster presentations: P12 hospice at home service listening, responding, developing. BMJ Supportive \& Palliative Care 2013;3:A13-A14.

10. Grady A, Travers E. Hospice at home 2: evaluating a crisis intervention service. Int J Palliat Nurs 2003;9:326-35.

11. Grande GE, Todd CJ, Barclay SI, et al. A randomized controlled trial of a hospital at home service for the terminally ill. Palliat Med 2000;14:375-85.

12. Jack $B$, Groves $K$, Baldry $C$, et al. Evaluation of the queenscourt at home pilot service. $2010 \mathrm{https} / / / \mathrm{www}$. researchgate.net/profile/B arbara_Jack/publication/267223274_Evaluation_of_the_Queenscour $\mathrm{t}$ at Home Pilot Service/links/551292420cf268a4aaea8a25.pdf (accessed 12 Sep 2017).

13. King G, Mackenzie J, Smith $\mathrm{H}$, et al. Dying at home: evaluation of a hospice rapid-response service. Int J Palliat Nurs 2000;6:280-7.

14. Koffman J, Higginson I, Naysmith A. Hospice at home--a new service for patients with advanced HIV/AIDS: a pilot evaluation of referrals and outcomes. Br J Gen Pract 1996;46:539-40.

15. Lucas B, Small N, Greasley P, et al. A reassuring presence: an evaluation of Bradford District Hospice at Home service. BMC Palliat Care 2008; $7: 9$

16. McLaughlin D, Sullivan K, Hasson F. Hospice at home service: the carer's perspective. Support Care Cancer 2007;15:163-70.

17. Payne S, Seymour J, Ingleton C, et al. Independent evaluation of the Marie Curie cancer care 'Delivering Choice Programme': International Observatory on End of Life Care Institute for Health Research, Lancaster University, 2008.

18. Purdy S, Lasseter G, Griffin T, et al. Impact of the Marie Curie Cancer Care Delivering Choice Programme in Somerset and North Somerset on place of death and hospital usage: a retrospective cohort study. BMJ Support Palliat Care 2015;5:34-9.

19. Savage L. St Giles Hospice: patient and carer satisfaction evaluation hospice @ home. 2012 http://www.stgileshospice.com/documen ts/19311Report-PatientandCarerSatisfaction.pdf (accessed 12 Sep 2017).

20. Sullivan KA, McLaughlin D, Hasson F. Exploring district nurses' experience of a hospice at home service. Br J Community Nurs 2013;10:11.

21. Tyrer F, Exley C. Receiving care at home at end of life: characteristics of patients receiving Hospice at Home care. Fam Pract 2005;22:644-6.

22. Whitmore S. Katharine house at home: developing a hospice at home team. Poster presentation at the Help the Hospices conference. 2012 http://www.helpthehospices.org.uk/our-services/education-training/ help-the-hospices-conference-2012/posters/A-innovative-approachhospice-at-home-team (accessed 12 Sep 2014).

23. Wye L, Lasseter G, Percival J, et al. Independent evaluation of the Marie Curie cancer care delivering choice programme in Somerset and North Somerset. Final Report. 2012 https://www.researchg ate.net/profile/Bethany_Simmonds2/publication/261098565_Delive ring_Choice_Proramme_in_Somerset_and_North_Somerset_Final_R eport/links/00b7d533314d41e272000000.pdf (accessed 12 Sep 2017)

24. Gage H, Holdsworth LM, Flannery C, et al. Impact of a hospice rapid response service on preferred place of death, and costs. BMC Palliat Care 2015;14:75

25. Holdsworth LM, Gage H, Coulton S, et al. A quasi-experimental controlled evaluation of the impact of a hospice rapid response community service for end-of-life care on achievement of preferred place of death. Palliat Med 2015;29:817-25.

26. Jack BA, O'Brien MR, Scrutton J, et al. Supporting family carers providing end-of-life home care: a qualitative study on the impact of a hospice at home service. J Clin Nurs 2015;24:131-40.

27. Jack BA, Mitchell TK, Cope LC, et al. Supporting older people with cancer and life-limiting conditions dying at home: a qualitative study of patient and family caregiver experiences of Hospice at Home care. $J$ Adv Nurs 2016;72:2162-72.

28. Spencer L. Hospice to home: a patient-centric multi professional community approach. Int J Palliat Nurs 2015;21:245-50.

29. Taylor K, Ali S, Bunting M. The palliative care partnership: the story so far. BMJ Supportive \& Palliative Care 2016;6(Suppl1):A1-A112.

30. Wilson J, Steers J, Steele K, et al. Service evaluation of community based palliative care and a hospice-at-home service. BMJ End of Life Journal 2015:0:1-11.

31. Woodyatt N, Singh S. Championing specialist care at home in everyday dying. BMJ Supportive \& Palliative Care 2016;6(Suppl1):A1-A112.

32. Bell G, Greene K, Hunt J, et al. Developing national standards for hospice-at-home services. End of Life Journal 2013;3:1-5

33. Richardson $\mathrm{H}$, Thomson A. Feedback on the survey of hospice at home: Help the Hospices. Oral Presentation, 2012.

34. PeolcPSP. Palliative and end of life care Priority Setting Partnership (PeolcPSP): putting patients, carers and clinicians at the heart of palliative and end of life care research. Final Report. $2015 \mathrm{http} / / /$ www.palliativecarepsp.org.uk/wp-content/uploads/2015/01/ PeolcPSP Final Report.pdf (accessed 3 Feb 2015)

35. Gomes B, Higginson IJ, Calanzani N, et al. Preferences for place of death if faced with advanced cancer: a population survey in England, Flanders, Germany, Italy, the Netherlands, Portugal and Spain. Ann Oncol 2012;23:2006-15

36. Sheppherd S, Wee B, Straus SE. Hospital at home: home-based end of life care. Cochrane Database Syst Rev 2011;7:CD009231.

37. Gomes B, Calanzani N, Gysels M, et al. Heterogeneity and changes in preferences for dying at home: a systematic review. BMC Palliat Care 2013;12:7.

38. Higginson IJ, Sarmento VP, Calanzani N, et al. Dying at home--is it better: a narrative appraisal of the state of the science. Palliat Med 2013;27:918-24.

39. Sleeman KE, Davies JM, Verne J, et al. The changing demographics of inpatient hospice death: Population-based cross-sectional study in England, 1993-2012. Palliat Med 2016;30:45-53.

40. Parliamentary \& Health Service Ombudsman. Dying without dignity: investigations by the Parliamentary and Health Service Ombudsman into complaints about end of life care. $2015 \mathrm{http} / / /$ www.ombudsman org.uk/_data/assets/pdf_file/0019/32167/Dying_without_dignity report.pdf downloaded 23/06/2015 (accessed 3 Feb 2017).

41. Pawson R, Tilley N. Realistic evaluation. 2nd edn. London: Sage, 2001

42. Pawson R, Tilley N. Realist evaluation. 2004 http://www. communitymatters.com.au/RE_chapter.pdf (accessed 3 Feb 2015).

43. Marchal B, Dedzo M, Kegels G. A realist evaluation of the management of a well-performing regional hospital in Ghana. BMC Health Serv Res 2010;10:24.

44. Wye L, Lasseter G, Percival J, et al. What works in 'real life' to facilitate home deaths and fewer hospital admissions for those at end of life?: results from a realist evaluation of new palliative care services in two English counties. BMC Palliat Care 2014:13:37.

45. Bhaskar R. A realist theory of science. Harvester Press: Sussex 1978.

46. Porter SR. In: Miller RL, Brewer JD, eds. The $A-Z$ of social research. London: Sage, 2003:256-9.

47. Stosz L. Literature review of the evidence base for a hospice at home service. Centre for Health Services Studies, University of Kent: Canterbury, 2008.

48. Marinetto M. Studies of the policy process: a case analysis. London: Prentice Hall Europe, 1998.

49. Rycroft-Malone J, Fontenla M, Bick D, et al. A realistic evaluation: the case of protocol-based care. Implement Sci 2010;5:38.

50. May CR, Finch T, Ballini L, et al. Evaluating complex interventions and health technologies using normalization process theory: development of a simplified approach and web-enabled toolkit. BMC Health Serv Res 2011;11:245.

51. Calanzani N, Higginson IJ, Gomes B. Current and future needs for hospice care: an evidence-based report. London: Help the Hospices (Help The Hospices Commission into the future of hospice care), 2013.

52. Wilson $\mathrm{P}$, Mathie $\mathrm{E}$, Keenan J, et al. Research with patient and public involvement: a realist evaluation. The RAPPORT study. Health services and delivery research 2015. Southampton (UK): NIHR Journals Library, 2015.

53. Curtis JR, Patrick DL, Engelberg RA, et al. A measure of the quality of dying and death. Initial validation using after-death interviews with family members. J Pain Symptom Manage 2002;24:17-31. 
54. Albers G, Echteld MA, de Vet HC, et al. Evaluation of quality-of-life measures for use in palliative care: a systematic review. Palliat Med 2010;24:17-37.

55. Hales S, Zimmermann C, Rodin G. Review: the quality of dying and death: a systematic review of measures. Palliat Med 2010;24:127-44.

56. Office of National Statistics. National Survey of Bereaved People (VOICES) 2013. Statistical Bulletin 2014. http://www.ons.gov.uk/ons/ dcp171778_370472.pdf (accessed 3 Feb 2015).

57. Guerriere DN, Coyte PC. The ambulatory and home care record: a methodological framework for economic analyses in end-of-life care. $J$ Aging Res 2011;2011:1-11.

58. Palliative care Outcome Scale. Integrated Palliative Outcome Scale (IPOS). 2012 http://pos-pal.org/ (accessed 3 Feb 2015).

59. Public Health England. National end of life care intelligence network palliative care clinical data set Guidance and definitions V1.0, 2016.

60. Stone K, Hotopf M, Koffman J, et al. Morecare capacity: mental capacity and processes of consent for research on end-of-life care. BMJ Support Palliat Care 2013;3:128.1-128.

61. U.S. National Library of Medicine. CRT hydration in the last days of life (Feasibility Study). 2015 https://clinicaltrials.gov/ct2/show/ NCT02344927 (accessed 26 Jun 2015).

62. Hales S, Chiu A, Husain A, et al. The quality of dying and death in cancer and its relationship to palliative care and place of death. J Pain Symptom Manage 2014;48:839-51.
63. Clark K, Cain J, Campbell L, et al. Caring for people dying in acute hospitals: a mixed-methods study to examine relative's perceptions of care. Palliat Support Care 2015;13:335-43.

64. Lewis-Newby M, Curtis JR, Martin DP, et al. Measuring family satisfaction with care and quality of dying in the intensive care unit: does patient age matter? J Palliat Med 2011;14:1284-90.

65. Oliver P, Jupp V. Purposive sampling. In: The SAGE dictionary of social research methods: Sage, 2006:244-5.

66. Gardiner C, Brereton L, Frey R, et al. Approaches to capturing the financial cost of family care-giving within a palliative care context: a systematic review. Health Soc Care Community 2016;24:519-31.

67. Curtis L. Unit costs of health and social care: University of Kent, 2014.

68. McEvoy R, Ballini L, Maltoni S, et al. A qualitative systematic review of studies using the normalization process theory to research implementation processes. Implement Sci 2014;9:2.

69. Rycroft-Malone J, Wilkinson J, Burton CR, et al. Collaborative action around implementation in collaborations for leadership in applied health research and care: towards a programme theory. $J$ Health Serv Res Policy 2013;18:13-26.

70. Stanfield RB. The workshop book: from individual creativity to group action. Cabriola Island, Canada: New Society Publishers, 2002.

71. Hewitt G, Sims S, Harris R. The realist approach to evaluation research: an introduction. Int J Ther Rehabil 2012;19:250-9. 